\title{
ANÁLISE E RECONHECIMENTO DE DOR EM IMAGENS 2D FRONTAIS DE RECÉM-NASCIDOS A TERMO E SAUDÁVEIS
}

\author{
Gilberto F. Teruel $^{1}$, Tatiany M. Heiderich ${ }^{2}$, Ruth Guinsburg ${ }^{2}$, Carlos E. Thomaz $^{1}$ \\ ${ }^{1}$ Centro Universitário FEI - São Bernardo do Campo, SP, Brazil \\ ${ }^{2}$ Universidade Federal de São Paulo (UNIFESP) - São Paulo, SP, Brazil \\ \{gilberto.ferreira.teruel, tatianyms, ruth.guinsburg\} @gmail.com \\ cetefei.edu.br
}

\begin{abstract}
Resumo. Esse artigo propõe uma sequência de procedimentos computacionais para detecção, interpretação e classificação de padrões em imagens bidimensionais frontais de faces para reconhecimento automático de dor em recémnascidos. Usando transformações de dados e extrações de características estatísticas de um banco de imagens reais de recém-nascidos a termo e saudáveis, foi possível abstrair e modelar a subjetividade dos profissionais de saúde treinados, quantificando o conhecimento humano na tarefa de reconhecimento de dor permitindo uma identificação automática. Tais resultados foram comparados com classificações das mesmas imagens, com base na escala de dor NFCS, pelos mesmos profissionais.
\end{abstract}

\begin{abstract}
This paper proposes a sequence of computational procedures for detecting, interpreting and classifying patterns in frontal two-dimensional images of faces for automatic recognition of pain in newborns. Using data transformation and extraction of statistical characteristics from a real-life, healthy-term newborn image database, it was possible to interpret and model the subjectivity of trained health professionals, quantifying human knowledge in the task of recognizing pain enabling automatic identification. These results were compared with NFCS based classifications by the same professionals of the same images.
\end{abstract}

\section{INTRODUÇÃO}

A percepção da dor é intrínseca a qualquer ser vivo que possui sistema nervoso central [Anand and Craig 1996]. Para seres humanos adultos, a dor pode ser identificada por meio de expressões verbais e indicações subjetivas. No entando, em lactentes pré-verbais existe uma dificuldade maior em identificar a dor e intensidade que a mesma se manifesta, devido à impossibilidade de comunicação verbal. Sendo assim, a identificação da dor se dá por meio da análise de ações e reações, não necessariamente objetivas, do recémnascido frente ao estímulo desagradável ou doloroso [Guinsburg and Cuenca 2010].

As consequências de experiências dolorosas em recém-nascidos são mais severas dado a imaturidade neurológica, assim como o uso irregular de analgesia ou sedação para controle da dor. Essas consequências tornam os recém-nascidos mais sensíveis e susceptíveis a experiências estressantes e potencialmente dolorosas, que podem propiciar impacto 
negativo na evolução neurológica, cognitiva e psiquiátrica deste indivíduo a curto e médio prazos [Grunau 2013, Guinsburg 1999, Chermont et al. 2003, Heiderich et al. 2015, Aymar and Coutinho. 2008]. Em consequência, diversas escalas para identificação de dor com métodos não-invasivos têm sido desenvolvidas, baseadas em observações de comportamento, como movimentos faciais, podendo ou não serem mescladas com indicadores fisiológicos [Lawrence et al. 1993, Stevens et al. 1996, Grunau and Craig 1987, Krechel and Bildner 1995]. Essas escalas e métodos possibilitam a supervisão de recémnascido em um período de tempo maior, através da criação de métodos de supervisionamento automático [Heiderich et al. 2015], auxiliando não somente a identificação mais precoce, como também uma compreensão melhor desta experiência negativa.

\section{MATERIAIS E MÉTODOS}

\subsection{Banco de imagens}

O banco de imagens de recém-nascidos contém imagens capturadas durante procedimentos potencialmente dolorosos e/ou estressantes necessários e aplicados aos recémnascidos. Esse banco é composto por 360 amostras de imagens de 30 recém-nascidos diferentes, com idade gestacional entre 34 e 41 semanas e entre 24 e 168 horas de vida (prematuros tardios ou a termo), alimentados e saudáveis [Heiderich et al. 2015].

As imagens foram dispostas de maneira aleatória e avaliadas por profissionais treinados da área da saúde com especialização em neonatologia, classificando a imagem como "Sem Dor"ou "Com Dor"de forma subjetiva e também com base na escala de dor NFCS - Neonatal Facial Coding System [Grunau and Craig 1987].

\subsection{Metodologia}

A metodologia proposta é composta das seguintes etapas principais:pré-processamento, com segmentação de faces e redimensionamento; marcação dos pontos fiduciais; normalização espacial das imagens, utilizando transformações não-lineares; construção do Atlas de Referência Inicial e Atlas de Recém-Nascidos e análise estatística multivariada, usando PCA (Principal Component Analysis), para encontrar as componentes principais cujos dados apresentam maior variância em relação aos conjuntos "Com Dor"e "Sem Dor", além de reduzir a dimensionalidade da informação, e LDA (Linear Discriminant Analysis), para encontrar a característica mais discriminante e classificar as imagens em escala contínua entre "Sem Dor"e "Com Dor".

\subsubsection{Pré-Processamento}

Para analisar e comparar as características entre imagens, foi utilizado a técnica HOG - histograma de gradientes orientados em conjunto com um classificador linear treinado SVM - Support Vector Machine para identificar e extrair a região da imagem que contém a face do recém-nascido. Esses métodos em conjunto permitem a identificação dos locais das faces, mesmo em imagens com ruído, artefatos e baixa/alta luminância [Dalal and Triggs 2005]. 


\subsubsection{Marcação dos Pontos Fiduciais}

Para normalizar espacialmente as imagens utiliza-se um algoritmo baseado em regressores em cascata [Kazemi and Sullivan 2014], o qual permite estimar a posição dos pontos fiduciais em faces de seres humanos presentes em imagens bidimensionais. Os regressores atuam de forma a contribuir para encontrar a posição esperada dos pontos fiduciais, gerando uma marcação automática de 68 pontos que descrevem o rosto do recém-nascido da imagem, preservando as características a serem estudadas.

\subsubsection{Normalização Espacial das Imagens}

O objetivo dessa etapa é alinhar espacialmente as características a serem comparadas e analisadas através de transformações rígidas (AFIM) e não-rígidas (FFD-Free Form Deformation), reduzindo as diferenças devidas a orientação espacial, escala, posição e diferenças nas formas das faces [Rueckert et al. 1999].

\subsubsection{Extração das Características Mais Expressivas}

Aqui as variáveis a serem analisadas e correlacionadas são os pixels das faces nas imagens do banco. Para encontrar as características que mais variam ou discriminam o conjunto de imagens utiliza-se a técnica PCA - Análise de Componentes Principais [Fukunaga 1990].

Nesse estudo o PCA foi utilizado a fim de analisar as características mais expressivas com maiores variâncias, sendo possível identificar as expressões faciais mais relevantes. Vale ressaltar que calcular o PCA como etapa intermediaria é necessário quando a dimensionalidade do espaço das características que representa uma imagem é muito maior do que o número total de imagens da amostra de treinamento $(n>>N)$. Quando o contrário é verdade $(N>n)$, essa etapa é desnecessária [Xavier et al. 2015].

\subsubsection{Características Mais Discriminantes}

Para encontrar um hiperplano onde todas as características a serem analisadas estejam presentes e se alterem em conjunto e de forma contínua de uma classe para outra é necessário utilizar técnicas supervisionadas de análise multivariada estatística como o LDA - Análise Discriminante Linear, generalização da proposta original desenvolvida por Ronald A. Fisher [Fisher 1936].

O LDA foi utilizado aqui com o propósito de separar os conjuntos "Sem Dor"e "Com Dor", através de combinações lineares das variáveis que caracterizam cada classe, maximizando a distância entre classes e minimizando a dispersão (variabilidade) de dados dentro de cada classe. Para que o LDA seja aplicado com sucesso o critério de Fisher deve ser satisfeito. Dado o problema em questão, essa condição foi satisfeita utilizando-se uma extensão da técnica chamada MLDA - Maximum uncertainty LDA [Xavier et al. 2015]. Sendo assim, o procedimento para navegar nas dimensões mais discriminantes pode ser descrito matematicamente por: 


$$
\boldsymbol{y}=\overline{\boldsymbol{x}}+j \cdot \sigma_{i} \cdot \boldsymbol{P}_{p c a} \cdot \boldsymbol{w}_{m l d a},
$$

onde $\overline{\boldsymbol{x}}$ é a imagem média a ser navegada, $j \in\{-3,-2,-1,0,1,2,3\}, \sigma_{i}$ é o desvio padrão das amostras de cada grupo, $\boldsymbol{P}_{p c a}$ é a matriz de autovetores gerada pelo PCA e $\boldsymbol{w}_{m l d a}$ é o autovetor gerado pelo MLDA (calculado através das amostras de imagens projetadas no espaço reduzido do PCA, computado intermediariamente antes do MLDA) [Xavier et al. 2015].

\section{RESULTADOS}

Dada a falta de normalização, artefatos e outros ruídos do conjunto inicial de 360 imagens, foram utilizadas ao todo 263 imagens para treinamento do modelo, sendo 121 dessas classificadas pelos profissionais de saúde como "com dor"e 142 imagens "sem dor". Essas imagens passaram pela etapa de pré-processamento e normalização do tamanho da imagem para 200x200 pixels.

Após segmentação das faces e redimensionamento foi feito a marcação dos 68 pontos fiduciais, mapeando as principais regiões do rosto do recém-nascido para registro das imagens na etapa de normalização espacial.

Nesse ponto da metodologia proposta tem-se duas etapas. A primeira é referente a criação dos Atlas de Referência Inicial e Atlas de Recém-Nascidos, a segunda é o registro completo das imagens do banco em relação ao Atlas de Recém-Nascidos.

O Atlas de Referência inicial é criado a partir de algumas imagens alinhadas selecionadas aleatoriamente de cada classe, "Sem Dor"e "Com Dor", com o objetivo de servir de referência para alinhar o restante das imagens do banco e criar o Atlas de RecémNascidos. A partir da criação desse Atlas é possível construir o Atlas de Recém-Nascidos para cada classe, obtendo uma imagem de referência mais genérica e com as características de cada classe.

Nesse ponto as imagens estão prontas para serem registradas primeiramente através de transformação rígida (AFIM) e depois através de transformação rígida e não-rígida (AFIM + FFD), em relação ao Atlas de Recém Nascidos correlacionado a classe da imagem a ser registrada. Sendo então possível extrair as características mais expressivas, utilizando PCA e observar as componentes de maior variância. Como esperado, no resultado do PCA nem todas as componentes estavam relacionadas ao problema em estudo, a principal componente correspondeu a luminosidade e as demais apresentaram alterações na mímica facial dos recém-nascidos relacionadas a transição entre sem dor até com dor.

Utilizando o LDA foi possível criar um hiperplano único capaz de gerar imagens sintéticas e visualizar as alterações características da transição entre cada classe nas mímicas faciais, conforme na figura 1.

Por fim, os resultados foram relacionados com as classificações dos profissionais da área da saúde que se basearam na escala NFCS versus as classificações contínuas obtidas pelo MLDA na metodologia proposta, sendo possível verificar concordâncias e discordâncias entre as classificações. A região da fronteira entre as classificações "Sem Dor"e "Com Dor"(NFCS = 3) apresenta maior discordância, como pode ser visto na imagem 2. Acredita-se que essa incerteza ou discordância de classificação tenha origem na 


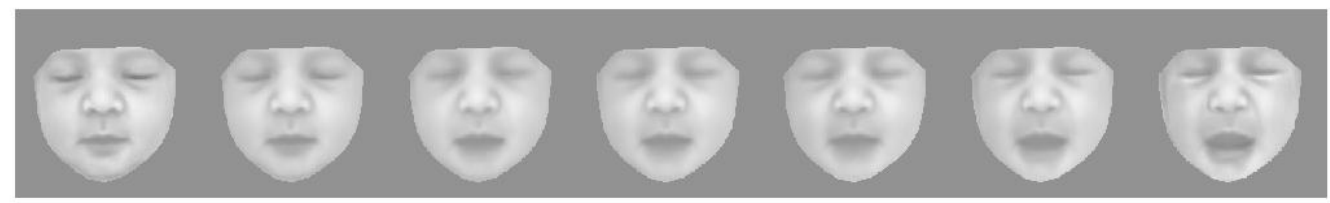

Figura 1. Características mais discriminantes, da esquerda para direita observase a evolução do estado sem dor para dor absoluta.

subjetividade e dificuldade de identificar com precisão as características exatas das amostras próximas a fronteira entre "Sem Dor"e "Com Dor". Além disso existem outros fatores como, por exemplo, o estresse, que acredita-se possuir algumas características similares a classificação "Com Dor", gerando maior dificuldade na classificação binária "Sem Dor"e "Com Dor".

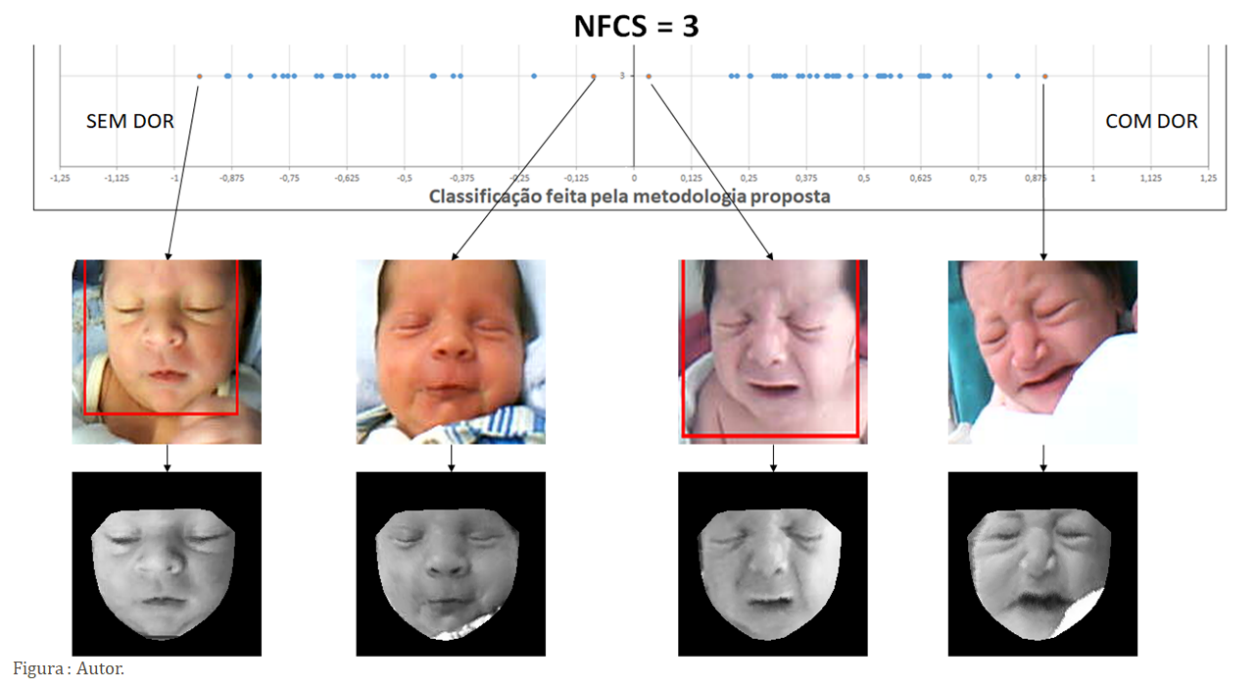

Figura 2. Relação entre a classificação das imagens pela escala NFCS com três características positivas e MLDA.

\section{CONCLUSÃO}

Na metodologia proposta foram utilizadas técnicas de pré-processamento e normalização de imagem, eliminando informações desnecessárias, segmentando faces e diminuindo desconformidades de escala, ângulo e morfologia facial. Essas técnicas foram essenciais para discriminar as imagens linearmente, permitindo classificações automáticas em espectros contínuos e possibilitando identificar o estado da dor do recém-nascido.

Os resultados apresentados no modelo possuem concordância em relação a classificação feita inicialmente por profissionais da área da saúde treinados para reconhecimento do fenômeno doloroso, permitindo uma melhor compreensão da subjetividade humana para essa tarefa de reconhecimento de dor. Já em relação a classificação da metodologia proposta versus a classificação dos mesmos profissionais utilizando NFCS, chegou-se a 72,77\% de acurácia. Acredita-se que essa discordância tenha origem em fatores de confusão não estudados aqui como, por exemplo, o estresse ou desconforto do recém-nascido. 


\section{Referências}

Anand, K. and Craig, K. (1996). New perspectives on the definition of pain. Pain, 67:3-6.

Aymar, C. and Coutinho., S. (2008). Fatores relacionados ao uso de analgesia sistêmica em neonatologia. Ver Bras Ter Intensiva, 20:405-415.

Chermont, A. G., Guinsburg, R., Balda, R. C., and Kopelman, B. I. (2003). O que os pediatras conhecem sobre avaliação e tratamento da dor no recém-nascido. Jornal Pediatr. (Rio J.), 79:265-272.

Dalal, N. and Triggs, B. (2005). Histograms of oriented gradients for human detection. In CVPR, pages 886-893.

Fisher, R. A. (1936). The use of multiple measurements in taxonomic problems. Annals of Eugenics, 7(7):179-188.

Fukunaga, K. (1990). Introduction to Statistical Pattern Recognition. Computer Science and Scientific Computing. Academic Press, Inc., 2nd edition.

Grunau, R. and Craig, K. (1987). Pain expression in neonates: facial action and cry. Pain, 28:395-410.

Grunau, R. E. (2013). Neonatal pain in very preterm infants: Long-term effects on brain,neurodevelopment and pain reactivity. Rambam Maimonides Med J., 25:4.

Guinsburg, R. (1999). Avaliação e tratamento da dor no recém-nascido. Jornal Pediatr. (Rio J.), 60:75-149.

Guinsburg, R. and Cuenca, M. (2010). A linguagem da dor no recém-nascido. Trends Genet., 1:1-5.

Heiderich, T. M., Leslie, A. T. F. S., and Guinsburg, R. (2015). Neonatal procedural pain can be assessed by computer software that has good sensitivity and specificity to detect facial movements. Acta Paediatrica, 104:63-69.

Kazemi, V. and Sullivan, J. (2014). One millisecond face alignment with an ensemble of regression trees. Proceedings of the 2014 IEEE Conference on Computer Vision and Pattern Recognition, pages 1867-1874.

Krechel, S. W. and Bildner, J. (1995). Cries: a new neonatal postoperative pain measurement score. initial testing of validity and reliability. Paediatr Anaesth, 5:53-61.

Lawrence, J., Alcock, D., McGrath, P., Kay, J., MacMurray, S., and Dulberg, C. (1993). The development of a tool to assess neonatal pain. Journal of Pain and Symptom Management, 6:59-66.

Rueckert, D., Sonoda, L. I., Hayes, C., Hill, D. L. G., Leach, M. O., and Hawkes, D. J. (1999). Nonrigid registration using free-form deformations: Application to breast $\mathrm{mr}$ images. IEEE Transactions on Medical Imaging, 18:712-721.

Stevens, B., Johnston, C., and Petryshen, P. (1996). Premature infant pain profile: development and initial validation. Research in Nursing Health, 12:13-22.

Xavier, I., Pereira, M., Giraldi, G., Gibson, S., Solomon, C., Rueckert, D., Gillies, D., and Thomaz., C. (2015). A photo-realistic generator of most expressive and discriminant changes in $2 \mathrm{~d}$ face images. In proceedings of the 6th International Conference on Emerging Security Technologies, EST-2015, 20:80-85. 